Supplement of Atmos. Chem. Phys., 21, 4709-4727, 2021

https://doi.org/10.5194/acp-21-4709-2021-supplement

(C) Author(s) 2021. CC BY 4.0 License.

(c) (1)

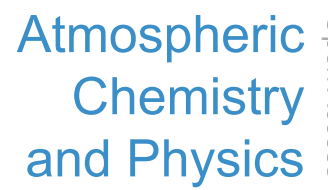

Atmospheric

and Physics

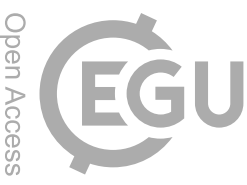

Supplement of

\title{
Pre-deliquescent water uptake in deposited nanoparticles observed with in situ ambient pressure $X$-ray photoelectron spectroscopy
}

\section{Jack J. Lin et al.}

Correspondence to: Nønne L. Prisle (nonne.prisle@oulu.fi) and Samuli Urpelainen (samuli.urpelainen@oulu.fi)

The copyright of individual parts of the supplement might differ from the article licence. 


\section{S1 Spectra fitting and uncertainty}

In this section, we explain the constraints used during the fitting of the measured XPS spectra. The peaks are represented with Voigt functions, or the convolution of a Lorentzian and a Gaussian shape. The peak shape has a Lorentzian contribution from the X-ray source, which uses the decay of $\mathrm{Al} \mathrm{K} \alpha$ and $\mathrm{Mg} \mathrm{K} \alpha$ core-holes to generate the X-rays. Physically, the Lorentzian shape represents the lifetime broadening effect experienced by a photoelectron. The Gaussian shape represents measurement uncertainties ${ }^{2}$. Other effects may induce asymmetric broadening, but an asymmetry parameter was usually not necessary to explain the peak shapes fitted here.

We also present the results of Monte Carlo error analysis of the fit results. The Monte Carlo analysis is done by creating a noiseless version of the fit and repeating the fitting procedure with random noise added to the noiseless spectrum. For the Monte Carlo analysis here, the fits were repeated either 100 or 200 times. We report the error in a given fit parameter as $\pm 2 \sigma$, where $\sigma$ is the standard deviation of the fit parameters from the set of Monte Carlo simulations.

\section{S1.1 NaCl}

Measurements were made on $\mathrm{NaCl}$ particles at UHV conditions as well as at 0 , 2 , and 5 mbar water vapor pressure inside the high pressure cell. Another set of measurements was taken at 0 mbar water vapor pressure after dosing with water vapor.

\section{S1.1.1 Na 1s}

Fit parameters for Na 1s spectra are shown in Table S1.

Table S1: Fitting parameters with uncertainty estimates from Monte Carlo simulations for $\mathrm{Na} 1 \mathrm{~s}$ spectra measured from $\mathrm{NaCl}$ particles.

\begin{tabular}{|c|c|c|c|c|c|c|c|c|}
\hline \multirow{2}{*}{$\begin{array}{l}\mathrm{P}_{\mathrm{H}_{2} \mathrm{O}} \\
\text { (mbar) }\end{array}$} & \multirow{2}{*}{$\begin{array}{c}\text { Peak } \\
\text { number }\end{array}$} & \multirow{2}{*}{$\begin{array}{c}\text { Binding } \\
\text { energy }(\mathrm{eV})\end{array}$} & \multicolumn{3}{|c|}{ FWHM $(\mathrm{eV})$} & \multirow{2}{*}{$\begin{array}{l}\text { Asym } \\
\text { metry }\end{array}$} & \multirow{2}{*}{$\begin{array}{l}\text { Area } \\
\text { (a.u.) }\end{array}$} & \multirow{2}{*}{$\begin{array}{c}\text { Monte Carlo } \\
\text { iterations }\end{array}$} \\
\hline & & & Lorentzian & Gaussian & Total & & & \\
\hline 0 & 1 & $1074.64 \pm 0.02$ & $570.99 \pm 175.70$ & $1709.38 \pm 135.07$ & $2034.93 \pm 221.62$ & $0 \pm 0$ & $4875.78 \pm 201.49$ & 200 \\
\hline 2 & 1 & $1072.42 \pm 0.05$ & $64.35 \pm 145.80$ & $1980.93 \pm 132.29$ & $2015.05 \pm 196.87$ & $0 \pm 0$ & $1022.05 \pm 63.74$ & 200 \\
\hline 5 & 1 & $1072.70 \pm 0.11$ & $1.28 \pm 0.01$ & $2714.72 \pm 911.72$ & $2714.75 \pm 911.72$ & $0 \pm 0$ & $217.08 \pm 78.77$ & 200 \\
\hline $0^{*}$ & $\begin{array}{l}1 \\
2\end{array}$ & $\begin{array}{l}1074.43 \pm 0.02 \\
1072.54 \pm 0.06\end{array}$ & $41.70 \pm 33.13$ & $2078.67 \pm 46.20$ & $2099.05 \pm 56.86$ & $0 \pm 0$ & $\begin{aligned} 3800.02 & \pm 101.13 \\
268.48 & \pm 22.61\end{aligned}$ & 200 \\
\hline
\end{tabular}




\section{S1.1.2 Cl 2p}

The $\mathrm{Cl} 2 \mathrm{p}$ spectra are split into two peaks from the $\mathrm{Cl} 2 \mathrm{p}_{1 / 2}$ and $\mathrm{Cl} 2 \mathrm{p}_{3 / 2}$ spin orbit components. The separation between the two is set to $1.6 \mathrm{eV}$, and the intensity of the $1 / 2$ component fixed to half that of the $3 / 2$ component. Both peaks are assumed to have the same line shape, meaning the peak area of the $1 / 2$ component is also half that of the $3 / 2$ component. Fit parameters for $\mathrm{Cl} 2 \mathrm{p}$ spectra are shown in Table S2.

Table S2: Fitting parameters with uncertainty estimates from Monte Carlo simulations for $\mathrm{Cl} 2 \mathrm{p}$ spectra measured from $\mathrm{NaCl}$ particles.

\begin{tabular}{|c|c|c|c|c|c|c|c|c|}
\hline \multirow{2}{*}{$\begin{array}{c}\mathrm{P}_{\mathrm{H}_{2} \mathrm{O}} \\
\text { (mbar) }\end{array}$} & \multirow{2}{*}{$\begin{array}{c}\text { Peak } \\
\text { number }\end{array}$} & \multirow{2}{*}{$\begin{array}{c}\text { Binding } \\
\text { energy }(\mathrm{eV})\end{array}$} & \multicolumn{3}{|c|}{ FWHM $(\mathrm{eV})$} & \multirow{2}{*}{$\begin{array}{l}\text { Asym } \\
\text { metry }\end{array}$} & \multirow{2}{*}{$\begin{array}{l}\text { Area } \\
\text { (a.u.) }\end{array}$} & \multirow{2}{*}{$\begin{array}{c}\text { Monte Carlo } \\
\text { iterations }\end{array}$} \\
\hline & & & Lorentzian & Gaussian & Total & & & \\
\hline 0 (UHV) & $\begin{array}{l}1 \\
2\end{array}$ & $\begin{array}{l}201.28 \pm 0.01 \\
202.88 \pm 0.01\end{array}$ & $455.45 \pm 7.80$ & $1203.56 \pm 11.32$ & $1465.30 \pm 13.74$ & $0 \pm 0$ & $\begin{array}{c}18580.90 \pm 118.06 \\
9290.45 \pm 59.03\end{array}$ & 100 \\
\hline 0 & $\begin{array}{l}1 \\
2\end{array}$ & $\begin{array}{l}201.41 \pm 0.03 \\
203.01 \pm 0.03\end{array}$ & $127.98 \pm 127.87$ & $1486.22 \pm 111.91$ & $1555.66 \pm 169.93$ & $0 \pm 0$ & $\begin{array}{r}1266.35 \pm 51.72 \\
633.18 \pm 25.86\end{array}$ & 100 \\
\hline 2 & $\begin{array}{l}1 \\
2\end{array}$ & $\begin{array}{l}199.27 \pm 0.01 \\
200.87 \pm 0.01\end{array}$ & $9.61 \pm 0.01$ & $1776.11 \pm 39.85$ & $1778.17 \pm 39.85$ & $0 \pm 0$ & $\begin{array}{l}632.06 \pm 13.99 \\
316.03 \pm 6.99\end{array}$ & 100 \\
\hline 5 & $\begin{array}{l}1 \\
2\end{array}$ & $\begin{array}{l}199.00 \pm 0.10 \\
200.60 \pm 0.10\end{array}$ & $4.64 \pm 24.57$ & $1416.94 \pm 141.16$ & $1417.57 \pm 143.29$ & $0 \pm 0$ & $\begin{array}{l}288.30 \pm 30.99 \\
144.15 \pm 19.68\end{array}$ & 100 \\
\hline $0^{*}$ & $\begin{array}{l}1 \\
2\end{array}$ & $\begin{array}{l}201.27 \pm 0.00 \\
202.87 \pm 0.00\end{array}$ & $214.68 \pm 2.99$ & $1388.00 \pm 19.23$ & $1506.21 \pm 19.47$ & $0 \pm 0$ & $\begin{array}{c}1084.50 \pm 13.46 \\
542.25 \pm 6.73\end{array}$ & 100 \\
\hline
\end{tabular}

\section{S1.2 Sucrose}

Sucrose particles were measured at $0,0.2,1$, and 5 mbar water vapor pressure.

\section{S1.2.1 C 1s}

Sucrose $\mathrm{C}$ 1s spectra contain three main peaks corresponding to distinct carbon atoms in C-C/C-H, C-O and O-C-O bonds. All peaks were forced to have the same symmetric Voigt line shape, as we expect the line shapes to be characterized by the natural broadening of the $\mathrm{Mg} K_{\alpha} \mathrm{X}$-ray emission lines $(680 \mathrm{meV})$ and the instrumental broadening of the electron analyzer (approximately $1000 \mathrm{meV}$ ). During fitting of the spectra, the separation between the C-O and O-C-O carbon peaks was fixed to $1.4 \mathrm{eV}$ at all UHV vacuum conditions below $10^{-8} \mathrm{mbar}$, based on previous XPS studies of disaccharides by Stevens and Schroeder ${ }^{3}$. The fitted $\mathrm{C}-\mathrm{O}$ and O-C-O peaks were, however, not linked to the C-C peak, as there are no purely C-C bound carbon in sucrose itself. The intensity ratios of the peaks were left unconstrained during fitting. Results of the fitting are shown in Table S3. 
Table S3: Fitting parameters with uncertainty estimates from Monte Carlo simulations for $\mathrm{C} 1 \mathrm{~s}$ spectra measured from sucrose particles.

\begin{tabular}{|c|c|c|c|c|c|c|c|c|}
\hline \multirow{2}{*}{$\begin{array}{c}\mathrm{P}_{\mathrm{H}_{2} \mathrm{O}} \\
\text { (mbar) }\end{array}$} & \multirow{2}{*}{$\begin{array}{c}\text { Peak } \\
\text { number }\end{array}$} & \multirow{2}{*}{$\begin{array}{c}\text { Binding } \\
\text { energy }(\mathrm{eV})\end{array}$} & \multicolumn{3}{|c|}{ FWHM $(\mathrm{eV})$} & \multirow{2}{*}{$\begin{array}{l}\text { Asym } \\
\text { metry }\end{array}$} & \multirow{2}{*}{$\begin{array}{l}\text { Area } \\
\text { (a.u.) }\end{array}$} & \multirow{2}{*}{$\begin{array}{c}\text { Monte Carlo } \\
\text { iterations }\end{array}$} \\
\hline & & & Lorentzian & Gaussian & Total & & & \\
\hline \multirow{3}{*}{0} & 1 & $285.90 \pm 0.00$ & \multirow{3}{*}{$1.16 \pm 0.00$} & \multirow{3}{*}{$2199.86 \pm 7.70$} & \multirow{3}{*}{$2199.89 \pm 7.70$} & \multirow{3}{*}{$0 \pm 0$} & $4207.45 \pm 14.71$ & \multirow{3}{*}{100} \\
\hline & 2 & $287.36 \pm 0.00$ & & & & & $728.57 \pm 2.56$ & \\
\hline & 3 & $288.76 \pm 0.00$ & & & & & $262.60 \pm 0.91$ & \\
\hline \multirow{3}{*}{0.2} & 1 & $285.65 \pm 0.02$ & \multirow{3}{*}{$234.81 \pm 102.91$} & \multirow{3}{*}{$1821.81 \pm 87.39$} & \multirow{3}{*}{$1950.42 \pm 135.01$} & \multirow{3}{*}{$0 \pm 0$} & $3734.51 \pm 112.12$ & \multirow{3}{*}{100} \\
\hline & 2 & $287.36 \pm 0.06$ & & & & & $607.31 \pm 24.92$ & \\
\hline & 3 & $288.76 \pm 0.06$ & & & & & $316.91 \pm 11.79$ & \\
\hline \multirow{3}{*}{1} & 1 & $285.57 \pm 0.04$ & \multirow{3}{*}{$223.93 \pm 198.68$} & \multirow{3}{*}{$1791.20 \pm 154.98$} & \multirow{3}{*}{$1913.74 \pm 251.98$} & \multirow{3}{*}{$0 \pm 0$} & $2745.13 \pm 139.91$ & \multirow{3}{*}{100} \\
\hline & 2 & $287.10 \pm 0.07$ & & & & & $416.77 \pm 19.78$ & \\
\hline & 3 & $288.50 \pm 0.07$ & & & & & $306.95 \pm 13.54$ & \\
\hline \multirow{3}{*}{5} & 1 & $285.57 \pm 0.08$ & \multirow{3}{*}{$1.96 \pm 2.29$} & \multirow{3}{*}{$1950.26 \pm 127.58$} & \multirow{3}{*}{$1950.35 \pm 127.60$} & \multirow{3}{*}{$0 \pm 0$} & $582.33 \pm 43.63$ & \multirow{3}{*}{100} \\
\hline & 2 & $287.56 \pm 0.09$ & & & & & $171.54 \pm 11.71$ & \\
\hline & 3 & $288.96 \pm 0.09$ & & & & & $78.24 \pm 5.06$ & \\
\hline
\end{tabular}

\section{S1.2.2 O $1 \mathrm{~s}$}

The sucrose $\mathrm{O}$ 1s spectra have two peaks arising from O-C-O and C-O components in the sucrose molecule. Both peaks were fixed to have the same symmetric line shape separated by $0.7 \mathrm{eV}$ that was fixed for all humidity conditions ${ }^{3}$. With the introduction of water vapor, a third peak appears and grows in intensity with increasing $\mathrm{RH}$. The separation between the two sucrose $\mathrm{C}-\mathrm{O}$ and $\mathrm{O}-\mathrm{C}-\mathrm{O}$ oxygen peaks was fixed at $0.7 \mathrm{eV}$ in all humidity conditions. Details of the fit results are shown in Table S4.

Table S4: Fitting parameters with uncertainty estimates from Monte Carlo simulations for $\mathrm{O} 1 \mathrm{~s}$ spectra measured from sucrose particles.

\begin{tabular}{|c|c|c|c|c|c|c|c|c|}
\hline \multirow{2}{*}{$\begin{array}{l}\mathrm{P}_{\mathrm{H}_{2} \mathrm{O}} \\
\text { (mbar) }\end{array}$} & \multirow{2}{*}{$\begin{array}{c}\text { Peak } \\
\text { number }\end{array}$} & \multirow{2}{*}{$\begin{array}{c}\text { Binding } \\
\text { energy (eV) }\end{array}$} & \multicolumn{3}{|c|}{ FWHM (eV) } & \multirow{2}{*}{$\begin{array}{l}\text { Asym } \\
\text { metry }\end{array}$} & \multirow{2}{*}{$\begin{array}{l}\text { Area } \\
\text { (a.u.) }\end{array}$} & \multirow{2}{*}{$\begin{array}{c}\text { Monte Carlo } \\
\text { iterations }\end{array}$} \\
\hline & & & Lorentzian & Gaussian & Total & & & \\
\hline 0 & 1 & $533.02 \pm 0.00$ & $7.78 \pm 0.07$ & $2209.60 \pm 14.72$ & $2210.74 \pm 14.73$ & $0 \pm 0$ & $1546.23 \pm 10.19$ & 100 \\
\hline 0.2 & $\begin{array}{l}1 \\
2 \\
3\end{array}$ & $\begin{array}{l}533.01 \pm 0.05 \\
533.71 \pm 0.05 \\
536.05 \pm 0.03\end{array}$ & $\begin{array}{c}0.02 \pm 0.63 \\
61.89 \pm 57.07\end{array}$ & $\begin{array}{c}2405.64 \pm 85.68 \\
1437.91 \pm 53.95\end{array}$ & $\begin{array}{c}2405.64 \pm 85.68 \\
1471.17 \pm 78.53\end{array}$ & $0 \pm 0$ & $\begin{aligned} 1819.54 & \pm 74.83 \\
796.45 & \pm 33.70 \\
1991.47 & \pm 84.85\end{aligned}$ & 100 \\
\hline 1 & $\begin{array}{l}1 \\
2 \\
3\end{array}$ & $\begin{array}{l}532.85 \pm 0.05 \\
533.55 \pm 0.05 \\
536.06 \pm 0.02\end{array}$ & $\begin{array}{c}0.08 \pm 0.40 \\
56.18 \pm 125.92\end{array}$ & $\begin{array}{c}2562.56 \pm 93.22 \\
1455.71 \pm 84.11\end{array}$ & $\begin{array}{r}2562.56 \pm 93.22 \\
1485.80 \pm 151.43\end{array}$ & $0 \pm 0$ & $\begin{array}{c}1491.24 \pm 62.35 \\
919.39 \pm 40.41 \\
4073.48 \pm 193.41\end{array}$ & 100 \\
\hline 5 & $\begin{array}{l}1 \\
2 \\
3\end{array}$ & $\begin{array}{l}532.45 \pm 0.12 \\
533.15 \pm 0.12 \\
536.03 \pm 0.01\end{array}$ & $\begin{array}{r}80.00 \pm 0.00 \\
518.36 \pm 30.49\end{array}$ & $\begin{array}{r}2500.00 \pm 0.00 \\
1206.97 \pm 34.41\end{array}$ & $\begin{array}{c}2542.37 \pm 0.00 \\
1507.75 \pm 45.98\end{array}$ & $0 \pm 0$ & $\begin{array}{c}277.42 \pm 16.56 \\
325.69 \pm 10.64 \\
8027.81 \pm 133.41\end{array}$ & 100 \\
\hline
\end{tabular}




\section{S1.3 Malonic acid}

Malonic acid particles were measured at $0,0.2$, and 1 mbar water vapor pressure. Due to time constraints, measurements at 0 mbar water vapor pressure after dosing was were not performed.

\section{S1.3.1 C $1 \mathrm{~s}$}

The malonic acid $\mathrm{C}$ 1s has three main peaks corresponding to $\mathrm{C}-\mathrm{C} / \mathrm{C}-\mathrm{H}$ bound carbon originating from adventitious carbon, $\mathrm{COOH}$ and $\mathrm{C}-\mathrm{C}$ carbon from malonic acid particles. The adventitious carbon $\mathrm{C}-\mathrm{C} / \mathrm{C}-\mathrm{H}$ and the malonic acid $\mathrm{COOH}$ peaks were not fixed in energy, whereas the malonic acid $\mathrm{C}-\mathrm{C}$ peak was fixed at $4.0 \mathrm{eV}$ lower binding energy than the $\mathrm{COOH}$ peak. The intensity of the attributed malonic acid C-C peak was fixed to half of that of the $\mathrm{COOH}$ peak, reflecting the expected stoichiometry in malonic acid molecules. The decomposition peaks DP1 and DP2 observed by Ferreira Jr. et al. ${ }^{1}$ appear in the 0 mbar spectrum and were fixed to be at $1.5 \mathrm{eV}$ (DP1) and $1.2 \mathrm{eV}$ (DP2) higher binding energies than the malonic acid $\mathrm{C}-\mathrm{C}$ and $\mathrm{COOH}$ carbon peaks, respectively. Upon exposure to water vapor, the DP2 feature disappears but a feature near the location of DP1 remains. This feature was let to vary freely in energy and intensity, but the shape was fixed to be the same as for the other lines in the spectra. The fitting results are shown in Table S5.

Table S5: Fitting parameters with uncertainty estimates from Monte Carlo simulations for $\mathrm{C}$ 1s spectra measured from malonic acid particles.

\begin{tabular}{|c|c|c|c|c|c|c|c|c|}
\hline \multirow{2}{*}{$\begin{array}{c}\mathrm{P}_{\mathrm{H}_{2} \mathrm{O}} \\
\text { (mbar) }\end{array}$} & \multirow{2}{*}{$\begin{array}{c}\text { Peak } \\
\text { number }\end{array}$} & \multirow{2}{*}{$\begin{array}{c}\text { Binding } \\
\text { energy }(\mathrm{eV})\end{array}$} & \multicolumn{3}{|c|}{ FWHM (eV) } & \multirow{2}{*}{$\begin{array}{l}\text { Asym } \\
\text { metry }\end{array}$} & \multirow{2}{*}{$\begin{array}{l}\text { Area } \\
\text { (a.u.) }\end{array}$} & \multirow{2}{*}{$\begin{array}{c}\text { Monte Carlo } \\
\text { iterations }\end{array}$} \\
\hline & & & Lorentzian & Gaussian & Total & & & \\
\hline \multirow{5}{*}{0} & 1 & $285.75 \pm 0.00$ & & & & \multirow{5}{*}{$0 \pm 0$} & $68477.98 \pm 49.32$ & \multirow{5}{*}{100} \\
\hline & 2 & $290.02 \pm 0.00$ & $674.72 \pm 0.49$ & $1525.08 \pm 1.16$ & $1917.57 \pm 1.27$ & & $13213.58 \pm 9.70$ & \\
\hline & 3 & $286.02 \pm 0.00$ & & & & & $6606.79 \pm 4.85$ & \\
\hline & 4 & $291.52 \pm 0.00$ & $609.88 \pm 0.00$ & $1980.96 \pm 0.00$ & $2326.98 \pm 0.00$ & & $2931.03 \pm 0.99$ & \\
\hline & 5 & $287.22 \pm 0.00$ & $609.88 \pm 0.00$ & $1500.00 \pm 0.00$ & $1852.31 \pm 0.00$ & & $1061.54 \pm 0.32$ & \\
\hline \multirow{4}{*}{0.2} & 1 & $285.48 \pm 0.00$ & \multirow{4}{*}{$512.75 \pm 1.18$} & \multirow{4}{*}{$1531.06 \pm 3.20$} & \multirow{4}{*}{$1823.45 \pm 3.41$} & \multirow{4}{*}{$0 \pm 0$} & $3513.38 \pm 7.58$ & \multirow{4}{*}{100} \\
\hline & 2 & $289.64 \pm 0.00$ & & & & & $443.65 \pm 0.96$ & \\
\hline & 3 & $285.64 \pm 0.00$ & & & & & $221.83 \pm 0.48$ & \\
\hline & 4 & $287.05 \pm 0.00$ & & & & & $402.31 \pm 0.85$ & \\
\hline \multirow{4}{*}{1} & 1 & $285.22 \pm 0.00$ & \multirow{4}{*}{$315.40 \pm 0.79$} & \multirow{4}{*}{$1498.03 \pm 5.75$} & \multirow{4}{*}{$1673.49 \pm 5.80$} & \multirow{4}{*}{$0 \pm 0$} & $2215.90 \pm 8.20$ & \multirow{4}{*}{100} \\
\hline & 2 & $289.07 \pm 0.00$ & & & & & $280.55 \pm 1.01$ & \\
\hline & 3 & $285.07 \pm 0.00$ & & & & & $140.27 \pm 0.51$ & \\
\hline & 4 & $286.50 \pm 0.00$ & & & & & $382.13 \pm 1.36$ & \\
\hline
\end{tabular}

\section{S1.3.2 O 1s}

Fit parameters for malonic acid O 1s spectra are shown in Table S6. 
Table S6: Fitting parameters with uncertainty estimates from Monte Carlo simulations for $\mathrm{O}$ 1s spectra measured from malonic acid particles.

\begin{tabular}{|c|c|c|c|c|c|c|c|c|}
\hline \multirow{2}{*}{$\begin{array}{l}\mathrm{P}_{\mathrm{H}_{2} \mathrm{O}} \\
\text { (mbar) }\end{array}$} & \multirow{2}{*}{$\begin{array}{c}\text { Peak } \\
\text { number }\end{array}$} & \multirow{2}{*}{$\begin{array}{c}\text { Binding } \\
\text { energy }(e V)\end{array}$} & \multicolumn{3}{|c|}{ FWHM $(\mathrm{eV})$} & \multirow{2}{*}{$\begin{array}{l}\text { Asym } \\
\text { metry }\end{array}$} & \multirow{2}{*}{$\begin{array}{l}\text { Area } \\
\text { (a.u.) }\end{array}$} & \multirow{2}{*}{$\begin{array}{c}\text { Monte Carlo } \\
\text { iterations }\end{array}$} \\
\hline & & & Lorentzian & Gaussian & Total & & & \\
\hline \multirow{6}{*}{0} & 1 & $532.33 \pm 0.00$ & & \multirow{6}{*}{$1485.39 \pm 12.77$} & & \multirow{4}{*}{$0 \pm 0$} & $23867.29 \pm 416.63$ & \multirow{6}{*}{200} \\
\hline & 2 & $533.28 \pm 0.00$ & $12045+821$ & & $155564+1518$ & & $17252.85 \pm 162.48$ & \\
\hline & 3 & $537.37 \pm 0.02$ & $129.45 \pm 8.21$ & & $1555.64 \pm 15.18$ & & $1271.58 \pm 5.83$ & \\
\hline & 4 & $538.33 \pm 0.02$ & & & & & $920.38 \pm 22.85$ & \\
\hline & 5 & $546.48 \pm 0.02$ & $3491.24 \pm 47.65$ & & $4146.51 \pm 49.34$ & $0.21 \pm 0.01$ & $46576.75 \pm 529.69$ & \\
\hline & 6 & $523.10 \pm 0.01$ & $1508.82 \pm 95.72$ & & $2997.23 \pm 96.57$ & $0.99 \pm 0.06$ & $7618.32 \pm 419.29$ & \\
\hline \multirow{5}{*}{0.2} & 1 & $532.07 \pm 0.01$ & & \multirow{5}{*}{$1381.57 \pm 23.65$} & & & $1752.00 \pm 30.48$ & \multirow{5}{*}{200} \\
\hline & 2 & $533.27 \pm 0.01$ & $129.45 \pm 8.21$ & & $1451.89 \pm 25.03$ & $0 \pm 0$ & $1386.19 \pm 23.44$ & \\
\hline & 3 & $535.78 \pm 0.02$ & & & & & $645.20 \pm 10.51$ & \\
\hline & 4 & $546.66 \pm 0.03$ & $3491.24 \pm 47.65$ & & $4079.17 \pm 53.20$ & $0.21 \pm 0.01$ & $2441.17 \pm 28.55$ & \\
\hline & 5 & $523.99 \pm 0.06$ & $5.68 \pm 0.36$ & & $1484.24 \pm 23.65$ & $-17.64 \pm 0.18$ & $229.39 \pm 3.43$ & \\
\hline \multirow{5}{*}{1} & 1 & $531.84 \pm 0.02$ & & \multirow{5}{*}{$1381.57 \pm 23.65$} & & & $1355.54 \pm 22.49$ & \multirow{5}{*}{200} \\
\hline & 2 & $533.04 \pm 0.02$ & $129.45 \pm 8.21$ & & $1451.89 \pm 25.03$ & $0 \pm 0$ & $1229.71 \pm 20.37$ & \\
\hline & 3 & $535.78 \pm 0.00$ & & & & & $3301.69 \pm 52.93$ & \\
\hline & 4 & $546.33 \pm 0.03$ & $3491.24 \pm 47.65$ & & $4079.17 \pm 53.20$ & $0.21 \pm 0.01$ & $1229.71 \pm 20.37$ & \\
\hline & 5 & $527.13 \pm 0.03$ & $1328.21 \pm 84.26$ & & $2224.08 \pm 87.52$ & $0 \quad \pm 0$ & $480.43 \pm 16.81$ & \\
\hline
\end{tabular}

\section{S2 Additional spectra from $\mathrm{NaCl}$}

\section{S2.1 Na 1s temperature dependence}

Measured $\mathrm{Na}$ 1s core level spectra from deposited $\mathrm{NaCl}$ nanoparticles at various temperatures are shown in Fig. S1. The spectra are all taken at 0 mbar water vapor pressure, both before and after sample was exposed to water vapor in the ambient pressure cell. 


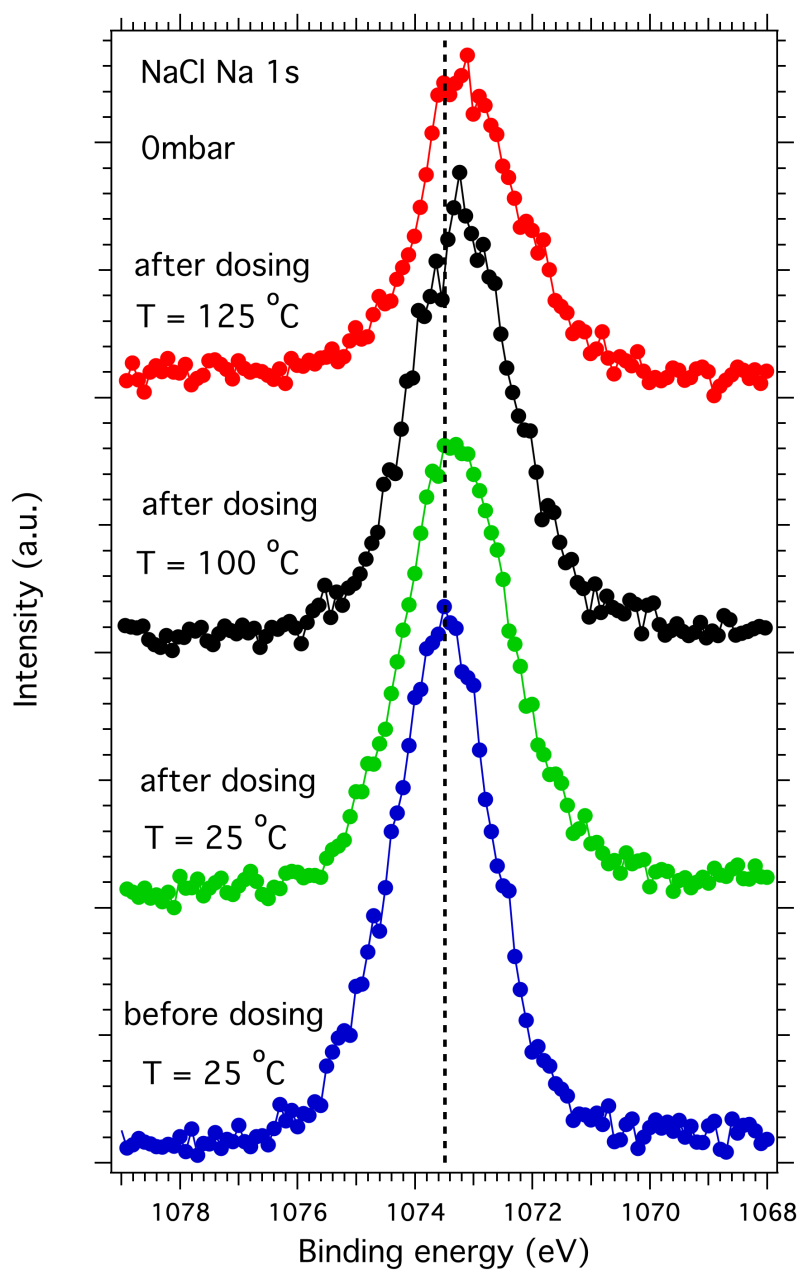

Figure S1: Change in $\mathrm{Na}$ 1s spectra of deposited $\mathrm{NaCl}$ aerosol particles with temperature. Photon energy was $1486.6 \mathrm{eV}$ from the $\mathrm{Al}$ anode. 


\section{S2.2 UHV spectra}

Spectra for $\mathrm{Na} 1 \mathrm{~s}$ and $\mathrm{Cl} 2 \mathrm{p}$ from $\mathrm{NaCl}$ particles measured at UHV conditions are shown in Fig. S2 and Fig. S3, respectively.

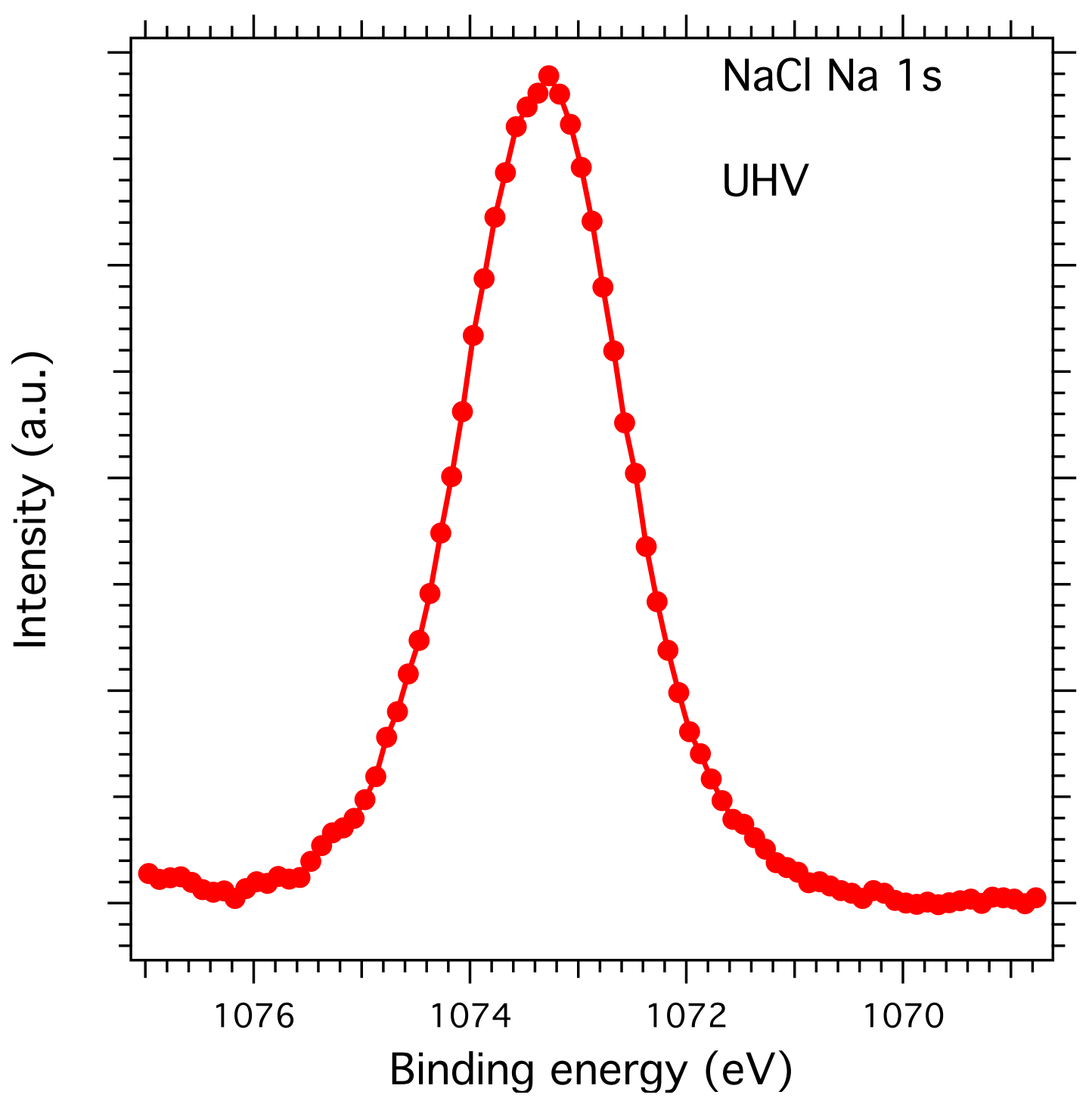

Figure S2: Na 1s spectrum measured at UHV conditions. Photon energy was $1486.6 \mathrm{eV}$ from the $\mathrm{Al}$ anode. 


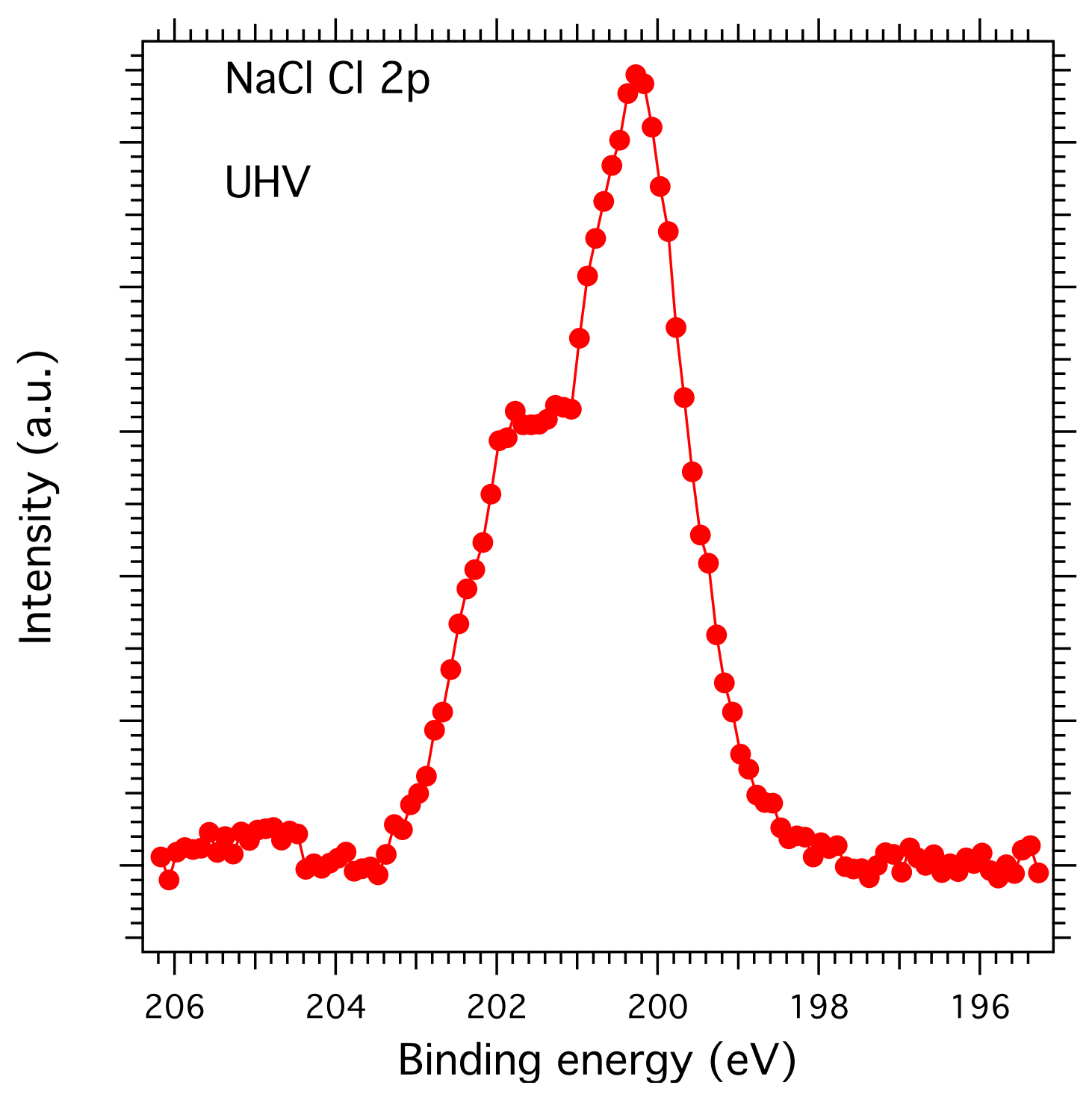

Figure S3: $\mathrm{Cl} 2 \mathrm{p}$ spectrum measured at UHV conditions. Photon energy was $1486.6 \mathrm{eV}$ from the $\mathrm{Al}$ anode. 


\section{References}

[1] Ferreira Jr., J. M., Trindade, G. F., Tshulu, R., Watts, J. F., and Baker, M. A.: Dicarboxylic acids analysed by x-ray photoelectron spectroscopy, Part I propanedioic acid anhydrous, Surface Science Spectra, 24, 011 101-8, 2017.

[2] Jain, V., Biesinger, M. C., and Linford, M. R.: The Gaussian-Lorentzian Sum, Product, and Convolution (Voigt) functions in the context of peak fitting $\mathrm{X}$-ray photoelectron spectroscopy (XPS) narrow scans, Applied Surface Science, 447, 548-553, 2018.

[3] Stevens, J. S. and Schroeder, S. L. M.: Quantitative analysis of saccharides by X-ray photoelectron spectroscopy, Surface and Interface Analysis, 41, 453462, 2009. 